\title{
AVALIAÇÃO DA POLÍTICA DE SAÚDE MENTAL A PARTIR DOS PROJETOS TERAPÊUTICOS DE CENTROS DE ATENÇÃO PSICOSSOCIAL ${ }^{1}$
}

\author{
Vanda Maria da Rosa Jardim², Maria do Horto Fontoura Cartana ${ }^{3}$, Luciane Prado Kantorski', André Luis \\ Alves de Quevedo ${ }^{5}$
}

\footnotetext{
${ }^{1}$ Recorte da pesquisa "Avaliação dos Centros de Atenção Psicossocial da região Sul do Brasil", com apoio financeiro do MCTCNPq e Ministério da Saúde.

${ }^{2}$ Doutora em Enfermagem. Professor Adjunto da Universidade Federal de Pelotas (UFPel). Rio Grande do Sul, Brasil. E-mail: phein@uol.com.br

${ }^{3}$ Doutora em Enfermagem. Professor Adjunto da Universidade Federal de Santa Catarina. Santa Catarina, Brasil. E-mail: horto@ccs.ufsc.br

${ }^{4}$ Doutora em Enfermagem. Professor Adjunto da Faculdade de Enfermagem e Obstetrícia da UFPel. Rio Grande do Sul, Brasil. E-mail: kantorski@uol.com.br

${ }^{5}$ Acadêmico do Curso de Enfermagem e Obstetrícia da UFPEL. Bolsista de Iniciação Científica do CNPq. Rio Grande do Sul, Brasil. E-mail: andrequevedo_sls@hotmail.com
}

RESUMO: Esta pesquisa teve como objetivo avaliar a política de saúde mental em sua concretização através da análise dos projetos terapêuticos de Centros de Atenção Psicossocial. Consiste numa pesquisa qualitativa, realizada em 2006 a partir de documentos fundamentais no processo de planejamento e gestão da política de saúde mental, a saber 21 projetos terapêuticos de Centros de Atenção Psicossocial da região Sul do Brasil que sofreram uma análise documental qualitativa por temas. Conclui apontando no conjunto de documentos analisados, propostas marcadamente alinhadas aos pressupostos da reforma psiquiátrica e outros que, para além das contradições típicas de um processo em construção, apresentam um discurso autoritário, disciplinador e em sentido contrário aos pressupostos, inclusive do aparato normativo do modo de atenção psicossocial.

DESCRITORES: Avaliação. Saúde mental. Políticas públicas.

\section{EVALUATION OF MENTAL HEALTH POLICY BASED ON PSYCHOSOCIAL CARE CENTER THERAPEUTIC PROJECTS}

\begin{abstract}
The objective of this study was to assess mental health policy in its implementation through analyzing Psychosocial Care Center therapeutic projects. It consists of a qualitative research, conducted in 2006 from key documents to the process of planning and managing mental health policy, namely 21 projects from therapeutic Psychosocial Care Centers of Southern Brazil which have undergone qualitative document analysis. It concludes pointing out proposals strongly aligned to the assumptions of psychiatric reform from the number of documents examined and others, which in addition to the typical contradictions of a process under construction, have an authoritarian, disciplining speech, contrary to assumptions, including those of the normative apparatus of the manner for psychosocial care.
\end{abstract}

DESCRIPTORS: Evaluation. Mental health. Public policies.

\section{ANÁLISIS DE LA POLÍTICA DE SALUD MENTAL A PARTIR DE LOS PROYECTOS TERAPÉUTICOS DE CENTROS DE ATENCIÓN PSICOSOCIAL}

\begin{abstract}
RESUMEN: Esta investigación tuvo como objetivo evaluar la política de salud mental a través del análisis de los proyectos terapéuticos de Centros de Atención Psicosocial. Se basa en una investigación cualitativa realizada en 2006, a partir de documentos fundamentales en el proceso de planificación y gestión de la política de salud mental, teniendo en cuenta veintiún proyectos terapéuticos de Centros de Atención Psicosocial de la región Sur de Brasil, los cuales fueron sometidos a un análisis documental cualitativo por temas. Se concluye que en el conjunto de documentos analizados, existen propuestas especialmente alineadas a los presupuestos de la reforma psiquiátrica, y otros que, más allá de las contradicciones habituales de un proceso en construcción, presentan un discurso autoritario, disciplinante y contrario, inclusive, a los presupuestos del aparato normativo del modo de atención Psicosocial.
\end{abstract}

DESCRIPTORES: Evaluación. Salud mental. Políticas públicas. 


\section{INTRODUÇÃO}

Os sofrimentos psíquicos, por suas magnitudes e impactos, têm se constituído como um dos principais problemas de saúde pública no mundo, implicando em sofrimento individual, familiar e social. Os transtornos neuropsiquiátricos representam aproximadamente 13\% dos Anos de Vida Ajustados por Incapacidade $(\mathrm{AVAD})^{*}$, chegando a atingir $33 \%$ ou quatro das seis primeiras causas de AVAD, quando se consideram todos os transtornos mentais, comportamentais e fatores relacionados. ${ }^{1-2}$

Frente a este contexto, são crescentes a preocupação e as discussões acerca dos saberes e fazeres em saúde mental dada sua magnitude e seus avanços teóricos e filosóficos no sentido de romper com o modelo clássico de exclusão.

A política, enquanto manifestação da organização do Estado através de um conjunto de objetivos e interesses materializados em ações que orientam a vida social, no caso específico da política de saúde, define a forma de organização da produção da atenção em saúde e determina as características de acesso, da qualidade da atenção e do processo de trabalho.

O universo das políticas de saúde produz um impacto importante e sua configuração atual, resultado da reforma produzida no setor, é decorrente de uma lógica diferenciada dos modelos tradicionais e, inclusive, aponta avanços na discussão que tradicionalmente a área de saúde coletiva promove sobre os princípios e diretrizes que norteiam o sistema. Este movimento acaba por tensionar alguns dos referenciais do Sistema Único de Saúde (SUS), reanimando o campo de discussão em saúde.

As políticas de saúde expressam uma dimensão de poder e se constituem em respostas de caráter social, através do movimento ou da omissão do Estado e suas representações frente às condições de saúde (individual e coletiva), implicando, assim, em relações de produção, distribuição, gestão e regulação de bens e serviços relacionados a saúde. ${ }^{3}$

Os diferentes discursos científicos de enfoque manicomial, da saúde mental, do sofrimento psíquico corroboraram a formulação e implementação de diferentes políticas de saúde. Destaca-se três diferentes discursos identificados ao longo da his- tória a partir do discurso manicomial, instituindo a vertente psiquiátrica e o gerenciamento científico da loucura. No período que se segue historicamente, sob influência da psicanálise, sociologia e antropologia, emerge o discurso da saúde mental, que encerra uma perspectiva biopsicossocial e renova a validação do gerenciamento científico da loucura. ${ }^{4}$ Somente no final da década de 80 , o discurso da desinstitucionalização, identificado com o paradigma psicossocial, vai defender a (des)construção epistemológica do sofrimento psíquico. ${ }^{5}$

No processo de concretização da política de saúde mental do país este artigo apresenta resultados da pesquisa que teve como objetivo avaliar a política de saúde mental em sua concretização através das políticas públicas considerando seus desdobramentos nos projetos terapêuticos dos Centros de Atenção Psicossocial (CAPS).

\section{METODOLOGIA}

O presente estudo foi realizado em $30 \mathrm{mu}$ nicípios dos três estados da região Sul do Brasil, cuja seleção respeitou a proporcionalidade de serviços por estado: três CAPS no Paraná; nove em Santa Catarina e 18 no Rio Grande do Sul. Os dados foram colhidos entre maio e julho de 2006. Neste artigo priorizou-se apresentar a avaliação da política de saúde mental a partir da análise qualitativa dos projetos terapêuticos (PTs) dos CAPS que integraram o estudo, considerando-se que este documento direciona as definições e implementações das diretrizes políticas que orientam a atenção em saúde mental nestes serviços e respectivos municípios. Constituindo-se em importante referência de planejamento e gestão da política de saúde mental no âmbito do município.

Do conjunto dos 30 CAPS estudados, sete CAPS não dispunham de projetos terapêuticos ou não os forneceram aos entrevistadores; dois projetos não foram analisados por indisponibilidade no momento da análise, totalizando assim 21 projetos terapêuticos analisados neste estudo. Para a análise documental qualitativa por temas desses projetos, foi construída uma tabela de registro dos recortes que indicavam as questões em discussão neste estudo, como também a análise inicial. A partir destes movimentos realizou-se uma sistematização das observações de análise o que permitiu a identificação dos pré-temas que orientaram uma

* AVAD é a medida de anos de vida ajustados em função de incapacidades e cada AVAD representa a perda de um ano de vida saudável, ou seja, se refere a tempo vivido com uma lesão ou tempo perdido por morte prematura, no qual se utiliza como referência a expectativa de vida. 
segunda leitura dos projetos terapêuticos e das tabelas de análise, com vistas a identificação dos temas e de sua descrição e análise crítica, no sentido de apontar as diferentes perspectivas adotadas, seus pontos de confluência e de conflito.

Os dados referentes às propostas municipais em saúde mental expressos nos projetos terapêuticos foram captados através de identificação de temas, ${ }^{6}$ incluindo a integralidade como tema central, nos aspectos relativos à rede de serviços considerando o acesso, acolhimento e intersetorialidade.

A presente pesquisa respeitou os procedimentos indicados na Declaração de Helsinki e das Resoluções No 196/96 e No 251/97 do Conselho Nacional de Saúde do Brasil, sendo apreciada e aprovada pelo Comitê de Ética em Pesquisa da Faculdade de Medicina da Universidade Federal de Pelotas, mediante Ofício $\mathrm{N}^{\mathrm{o}}$ 074/05 de 11 de novembro de 2005 e mantendo-se anonimato dos serviços.

\section{RESULTADOS E DISCUSSÃO}

Com vistas a conhecer a universo concreto dos CAPS, optou-se por analisar os Projetos Terapêuticos (PTs) destes serviços, que conforme a Portaria 147/1994, ${ }^{7}$ se constitui no conjunto de objetivos e ações, estabelecidos e executados pela equipe multiprofissional, voltados para a recuperação do paciente, desde a admissão até a alta. Incluindo o desenvolvimento de programas específicos e interdisciplinares, adequados à característica da clientela, visando compatibilizar a proposta de tratamento com a necessidade de cada usuário e de sua família. Envolve, ainda, a existência de um sistema de referência e contra-referência que permita o encaminhamento do paciente, após a alta, para a continuidade do tratamento. Representando assim a existência de uma filosofia que norteia e permeia todo o trabalho institucional.

Entre os 30 CAPS estudados, 15 são CAPSI e 15 são CAPSII. A distribuição seguindo a concentração de serviços resultou em um grupo selecionado aleatoriamente com a seguinte distribuição: um CAPSI no Paraná, cinco em Santa Catarina e nove no Rio Grande do Sul e dois CAPSII no Paraná, quatro em Santa Catarina e nove no Rio Grande do Sul.

Os projetos terapêuticos, apesar de previstos desde 1994 parecem ainda se constituir em elemento novo no universo dos centros de atenção psicossocial, especialmente quando se considera a indisponibilidade material dos PT's em alguns serviços (mais de 20\% dos CAPS estudados).
Em uma primeira análise foi possível identificar uma diversidade de opções teóricas e metodológicas na construção dos 21 projetos terapêuticos analisados. Entretanto, parece que via de regra, estes projetos são construídos por um ou mais técnicos com vistas ao cadastramento do serviço e em muitos locais este instrumento de planejamento e gestão não é incorporado ao cotidiano do serviço.

Neste conjunto de documentos é possível identificar propostas marcadamente alinhadas aos pressupostos da reforma psiquiátrica e outros que, para além das contradições típicas de um processo em construção, apresentam um discurso autoritário, disciplinador e em sentido contrário aos pressupostos, inclusive do aparato normativo. Assim como em um pequeno grupo, o projeto terapêutico se traduz pela reunião material de projetos de trabalho individual de diferentes profissionais de saúde.

As abordagens disciplinantes incluem desde o controle sobre tipos de vestimentas, comportamentos, controle espacial, até a impossibilidade de negociação de projetos terapêuticos individuais. Ao mesmo tempo desvelam uma dificuldade conceitual com princípios elementares do paradigma psicossocial, conforme pode ser evidenciado nos trechos.

[...] não concordando com o plano terapêutico o mesmo será referendado para a unidade básica de origem [...] (PT 8).

Normas internas em cada plano. Horários, proibições [...] (PT 21, PT 20, PT 30).

Não é permitido o uso de roupas justas, muito decotadas, minissaias [...] (PT 15).

[...] saúde mental envolve ações de preservar e recuperar aquilo que torna as pessoas humanas [...] evitando qualquer forma de castigo físico ou psicológico [...] (PT 8).

Os trechos destacados dos projetos terapêuticos estão em oposição ao paradigma psicossocial, ${ }^{5}$ no que se refere à participação do usuário que, nesta perspectiva, tem um papel ativo, na medida em que o sujeito da atenção é um indivíduo (orgânico, social e cultural) e na relação entre o serviço e o usuário que se sustenta na idéia de implicação subjetiva e sociocultural de um sujeito de desejo. Assim os aspectos autoritários parecem indicar dificuldades nos processos de negociação inerentes ao encontro das perspectivas do serviço e dos trabalhadores com os usuários.

Tais limites, em muitas situações observadas, parecem apontar muito mais a ausência de um suporte institucional qualificado do que uma 
opção teórica sedimentada. Inclusive porque em muitos projetos terapêuticos também se identifica claramente a busca de construção de uma perspectiva que inclua, que se constitua em um espaço de negociação, reconhecendo inclusive os conflitos que perpassam a relação entre as diferentes abordagens.

As assembléias [...] espaço privilegiado de discussão [...] lugar que possibilita o exercício da fala de cada um e da escuta do outro [...] grupo possui como característica ser aberto, dinâmico, com a presença de relações contraditórias, de amor édio, de alegria e tristeza, de liberdade e aprisionamento. Conflitos são analisados e discutidos, buscando o confronto com pensamentos e atitudes manicomiais entre os participantes [...] parte do imaginário social [...] passíveis de ser transinstitucionalizados [...] conversando, por vezes lutando [...] (PT 3).

No texto que descreve alguns aspectos das assembléias realizadas no CAPS (PT 3), é possível identificar um exercício de interlocução, no qual o saber técnico busca se diferenciar do poder coercitivo, ao mesmo tempo em que reconhece as dificuldades de romper com modelos institucionalizados e internalizados por uma histórica cultura de exclusão e de não reconhecimento da diferença e da singularidade, o que novamente nos faz retomar a descrição do paradigma psicossocial.

Em relação a rede de atenção em saúde mental, os projetos terapêuticos analisados indicam um conjunto de características e possibilidades que nos permitem conhecer acerca do desafio de articulação de ações e dispositivos utilizados.

Um primeiro aspecto identificado nos PTs se relaciona a idéia de acesso ao CAPS. O acesso é previsto, na grande maioria dos serviços utilizando a expressão acolhimento, entretanto, já em uma primeira leitura se percebe que existe diferentes perspectivas para o que e como fazer a acolhida dos usuários.

Acolher $100 \%$ da demanda que busca atendimento [...] no CAPS para avaliação [...] inclusão ou encaminhamento [...] (PT 1).

[...] acolhendo todos os usuários que procuram o serviço $[\ldots]($ PT 2).

Todas as pessoas são atendidas [...] escuta(PT 3).

Disponibilidade para o atendimento e/ou escuta imediata [...](PT 7).

Plantão semanal de acolhimento que ocorre [...] nas sextas-feiras de manhã [...] (PT 13).

A idéia de acolhimento assume diferentes sentidos no contexto analisado, o que também se observa na produção teórica acerca do tema.
Acolher se relaciona a humanização, escuta qualificada, resolubilidade e responsabilização frente às necessidades em saúde do usuário.

$\mathrm{O}$ acolhimento se refere a afirmação do princípio de universalidade em um lógica usuário-centrada em uma abordagem permeada de solidariedade. ${ }^{8}$ Em uma perspectiva diversa o acolhimento é orientado por quatro aspectos. ${ }^{9}$ São eles: o acesso em seus aspectos geográficos e organizacionais; a escuta como atitude dos trabalhadores de saúde em suas relações intra-equipe e com a população; a técnica, em seu desdobramento operacional do trabalho em equipe, saberes e práticas e, ainda a reorientação de serviços, enquanto projeto institucional.

De forma semelhante é possível identificar outros três aspectos que condicionam o acolhimento, e que inclui a postura e prática profissional; a gestão do processo de trabalho e a perspectiva apontada pela política de saúde. $\mathrm{O}$ acolhimento não pode ser confundido com uma triagem, mesmo que qualificada, ou ainda com uma escuta humanizada. ${ }^{10}$

A questão que se coloca aqui frente as abordagens apresentadas anteriormente é a perspectiva centrada no acesso universal, orientada pelas necessidades objetivas e subjetivas do usuário em oposição a uma perspectiva de atenção em saúde organizada em uma lógica espacial, via de regra, tomando como referência o local de moradia.

Entende-se que o acolhimento implica em uma orientação política que traz para dentro dos serviços e do sistema de saúde a tensão e os conflitos gerados pelas necessidades em saúde, modificando estruturas e processos da atenção em saúde.

Nos PTs, o acolhimento pode se relacionar a escuta, a triagem e inclusive ao que foi descrito como acolhimento realizado em apenas um turno por semana. Em um grande conjunto de CAPS a maioria dos trabalhadores de saúde parece estar envolvida com o acolhimento. Em poucos serviços isto se dá através de uma coordenação realizada por psicólogos, ou realizado especificamente pela assistente social ou ainda pelo técnico de enfermagem. Os dados também indicam que um segundo modelo, presente nos serviços, ocorre com uma triagem realizada por técnico de enfermagem e um "acolhimento" realizado por profissionais de nível superior, especialmente os psicólogos, em escala de plantão.

A forma de acesso aos serviços indica a convivência de dois modelos, praticamente em igual medida e extensão no conjunto de PTs anali- 
sados: um sistema de referência, típico do modelo triangular, com diferentes níveis de atenção e um modelo que se aproxima da lógica do círculo.

O modelo piramidal de atenção em saúde, com fluxos ascendentes e descendentes atende a uma lógica racionalizadora distanciada do movimento que os usuários realizam ao buscar os serviços de saúde. O modelo com múltiplas portas de entrada, além de acolher melhor o usuário romperia com a idéia de topo, presente no modelo triangular, que acaba por hierarquizar as relações de poder e importância na rede de atenção em saúde. Assim, o caráter do acolhimento está vinculado diretamente ao tipo de acesso e ao modelo de organização institucional.

Os trechos a seguir demonstram a presença tanto do modelo triangular, como do modelo de círculo, presente nos PTs dos CAPS da região sul do Brasil.

[...] paciente chega ao serviço através de procura espontânea ou encaminhamento de profissionais [...] (PT 11).

[...] usuário pode procurar diretamente o serviço ou por encaminhamento de qualquer serviço da saúde, preferencialmente da região em que reside [...] (PT 15).

[...] encaminhamentos assinados pelos profissionais médicos, psicólogos ou enfermeiro no PSF ou pronto atendimento $[. .$.$] (PT 24).$ (PT 25).

[...] serão atendidos [...] todos os que procurem.

A porta de entrada para o CAPS por três vias: Ambulatório; UBS e busca direta ao serviço em decorrência da falta de psiquiatra nas UBS e ambulatório (PT 29).

Esta divisão quanto à forma de acesso parece se relacionar com a idéia de porta de entrada do sistema, referida no conjunto normativo da atenção em saúde mental e ao mesmo tempo com a idéia de serviço de alta complexidade organizado e incluído nas ne.ociações dos planos regionais, indicados na Norma Operacional de Assistência a Saúde (NOAS).

Na maioria dos serviços a articulação se dá via Unidade Básica de Saúde tanto no sentido da referência como da contra-referência e também é neste espaço que os trabalhadores dos CAPS realizam, conforme os projetos terapêuticos, atividades de supervisão e capacitação, conforme indica a legislação em saúde mental. Alguns profissionais dos CAPS circulam em serviços da rede básica para atendimento da demanda.
O que se questiona é em que medida a realização de atendimentos favorece a articulação da rede ou dilui a proposta de trabalho do CAPS?

[...] descentralizar o atendimento em saúde mental, implantando e ampliando o atendimento em unidades básicas de saúde [...] supervisionar e capacitar as equipes de atenção básica, serviços e programas de saúde mental no âmbito do território [...] Atendimento psicológico de mais uma vez por semana no posto $x$ [...], y ez. [...] (PT 1).

Grupos operativos com a equipe da rede básica, com o objetivo de trocar experiências (PT 2).

[...] fazem parte da rede o PACS, unidades de saúde que se articulam e formam a rede de atenção á saúde mental. (PT 4).

Organizar uma rede de assistência pública hierarquizada, regionalizada e descentralizada, estabelecida sobre a rede básica que será provida por um sistema de referência e contra-referência, como forma de encaminhamento e informação entre as diversas unidades [...] (PT 15).

Supervisionar e capacitar as equipes de atenção básica, serviços e programas de saúde mental [...] (PT 28).

Os PTs têm poucas indicações da existência ou do tipo de relação dos CAPS com hospitais e outros serviços e ações previstos na rede de atenção em saúde mental. Em muitos momentos a própria idéia de articulação da rede é muito mais indicativa ou programática do que operacional e o item referência e contra-referência presente no modelo adotado de descrição dos PTs em algumas situações está vazio (de descrição) ou indica apenas a construção de um formulário de referência e contra-referência.

Identificou-se, em aproximadamente um terço dos PTs, uma descrição do número de leitos para internação psiquiátrica em hospital geral ou da realização de negociação para o credenciamento de leitos. Em um dos CAPS, os trabalhadores realizam supervisão, suporte e consultoria durante todo o período de internação em hospital geral. Também aparecem indicações de relações informais, inclusive aquelas em que a informalidade esta prevista formalmente.

Atendimento hospitalar [...] com encaminhamento para hospital $Y$ [geral] (PT 28).

Os atendimentos de urgência serão atendidos apenas sob encaminhamento diretamente com o profissional [...] do CAPS [pessoal ou telefônico] [...] (PT 24).

Caso o paciente necessite de internação, o contrato é feito junto a central de leitos ou diretamente com os hospitais [...](PT 15). 
Em referência e contra-referência: consiste em documentação específica em anexo documento de referência e contra-referência do CAPS (PT 11).

Para se estabelecer uma rede de referência e contra-referência é necessário mapear os locais que mantém vinculo direto ou indireto com o serviço, especificando quais os critérios de admissão em cada serviço, modalidades de atendimento, bem como estabelecer critérios para encaminhamento [...] serviço de menor complexidade deverá facilitar o encaminhamento, quando necessário, ao serviço de maior complexidade após cada abordagem de maior complexidade e de acordo com sua evolução [...] deverá ser encaminhado ao nível de menor complexidade para que não haja desvinculação do contexto onde vive, fortalecendo o vinculo anteriormente estabelecido [...] é muito importante o estabelecimento de um mapa de como ocorrem os encaminhamentos [...] deve ser conhecido por todos do serviço e pela coordenadoria regional de saúde [...](PT 7).

O processo de discussão com o hospital X [hospital geral] quanto ao cadas tro de leitos psiquiátricos em hospital geral [...] poderá colocar 6 leitos para urgências e emergências psiquiátricas (PT 6).

A discussão de rede de atenção em saúde mental identificada nos PTs dos serviços implica em alguns aspectos de forma de acesso, acolhimento, referência e contra-referência. Discute-se acesso, descentralização, capacitação, supervisão no contexto de rede. As indicações são mais sugestivas do que operacionais e, em geral incluem especificamente as ações (e os equipamentos) de saúde mental e a atenção básica. Poucas referências à existência de um suporte de urgência, emergência e hospital geral ou psiquiátrico.

Pensar uma rede de atenção as pessoas [...] no âmbito do SUS é um desafio que mexe com nossos desejos e utopias de enfrentar injustiças, inquietude e exclusão social [...] a rede traz em si um modelo de compartilhamento de recursos (PT 4).

Quando se inclui uma perspectiva de intersetorialidade, o que se percebe são objetivos orientados para uma idéia de territorialidade e intersubjetividade e a operacionalização e reprodução intramuros de um conjunto diversificado de ações, com poucas referências efetivas de ações que contemple outros serviços, ações e instituições.

Percebe-se uma marcada relação com instituições judiciais e policiais o que pode indicar a forte presença de uma cultura da sociedade de contenção e até criminalização do sofrimento psíquico.

As inter-relações mais estreitas se dão com o fórum, conselho tutelar e casa de atenção ao menor (PT 3).
A integração entre a rede de saúde mental com outros recursos comunitários é estratégico na promoção da saúde mental, tais como: promotoria pública, hospital geral, AAs, grupo X (atendimento a usuários de drogas), pastorais da saúde, criança; secretaria de educação, planejamento, desenvolvimento social, sociedades assistenciais, Conselho Municipal de Saúde, Conselho Tutelar, organizações comunitárias, universidades e outros, participação em conselhos regionais, municipais e com grupos de abordagens livres de preconceitos [...] (PT 6).

A intersetorialidade se constitui em uma compreensão ampliada do processo saúde-doença e das necessidades complexas das pessoas. A intersetorialidade se constitui em uma estratégia de articulação de recursos e implica necessariamente na negociação de propostas no horizonte político do território e das políticas públicas.

Identifica-se também uma idéia de rede que perpassa movimentos em conselhos, grupos, ações no contexto social, no sentido de construir uma intervenção ampliada para modificar ou intervir em um quadro de preconceitos sociais. Como também na perspectiva da intersetorialidade a idéia de integralidade se faz mais presente.

Em responsabilidades [...] integral pela atenção aos sujeitos, criando suportes para as diferentes necessidades da população [...] (PT 7).

Discussão com toda a sociedade, pode-se oferecer um programa de saúde mental [...] cujas parcerias, decisões e ações abranjam toda a comunidade (PT 15).

Há necessidade permanente de interlocução com a comunidade, através do aporte de intercâmbios que auxiliem [...] a desmistificação da doença mental (PT 17).

Em ações inter-setoriais: projetos: - De inserção no trabalho [...] capacitação e saúde do trabalhador; De alfabetização e adultos em parceria com secretaria da educação; - De inserção cultural em parceria com grupo de Hip-hop; - De inserção de lazer em parceria com entidades esportivas e outras: futebol, ingressos para cinema, teatro, eventos e entretenimento; - Produção de agendas para uso na secretaria de saúde em parceria; - Trabalho inter-setorial junto a moradores de rua; trabalho inter-setorial junto a população adulta em vulnerabilidade; - Atividades vinculadas ao ensino e pesquisa; - Em acolhimento: encaminhamento; - Território está em discussão [...] (PT 18).

O processo de construção de redes intersetoriais implica em um conjunto de dificuldades e ao mesmo tempo de possibilidades de intervenção em situações complexas, em ações coletivas dirigidas as diferentes e diversas nuances da realidade, pressupondo a integração de diferentes setores. ${ }^{12}$ 
A integralidade pressupõe uma ação intersetorial caracterizada pela construção compartilhada e coletiva de referenciais em saúde, materializadas em dado território, ${ }^{13}$ ou seja, a materialização de possibilidades frente a identificação e co-responsabilização dos sujeitos sociais.

A grande dificuldade que ainda se observa, parece se relacionar as dificuldades de compreensão da interface política que esta diretriz assume, especialmente no contexto das políticas públicas e da dificuldade da gestão, dos serviços e dos trabalhadores de saúde em romper com práticas orientadas pela centralidade da saúde, como campo definidor inquestionável de práticas.

\section{CONCLUSÃO}

A construção da análise apresentada neste artigo se constitui em uma leitura de um universo rico de possibilidades, de tensões e conflitos. E estas condições não se dão necessariamente em razão do sofrimento psíquico, mas da diversidade de intenções que permeiam muitos discursos e práticas da saúde mental. Uma intenção que em dado momento se cristaliza na ousadia de trabalhadores, usuários e familiares, no sentido de viver a possibilidade e inevitabilidade da diferença. E, ao mesmo tempo também apresenta amarras mantidas por uma tradição autoritária da mesma saúde mental, das práticas de saúde em geral e do modelo de organização social que historicamente organiza a vida e as relações cotidianas. Estes movimentos se dão através do esquadrinhamento, classificação e exclusão de quaisquer ações, reações ou sofrimentos que em suas interfaces tensionem a lógica de adequação a este modelo.

O movimento de reforma psiquiátrica, no contexto das políticas públicas de saúde tem oferecido, ao longo dos últimos vinte anos, elementos que tensionam os interesses econômicos em saúde e que também sofrem suas influências, na medida em que se concentra nas portas de entrada do sistema, atingindo ainda pouco os hospitais privados ou outros serviços alinhados a interesses privados.

Os conflitos de interesses acabam por produzir contradições na política e em seu processo de concretização. Entre eles ainda os enquadramentos sociais do sofredor psíquico nos moldes da moral e do mercado de trabalho, seja por dificuldades de concretizar uma idéia de territorialização concreta e subjetiva das pessoas ou ainda pela histórica constituição de grupos impregnados pela exclusão. O que estaria por trás ou o risco que tais práticas representam é uma medicalização social, uma tentativa de romper com a lógica de normalidade, mas que encontra na forma de organização social da vida e do trabalho contradições vividas e consumidas através de discursos de humanização, do trabalho como redentor e da disciplina como forma de correção do desvio através da coerção.

$\mathrm{O}$ valor da disciplina presente em muitos PTs é uma dos grandes desafios. Não é à toa que a burguesia liberal pode suportar uma mudança de paradigma no tratamento do sofrimento psíquico. Ela anteviu, em alguma medida que seria possível conter os gastos públicos diretos em saúde mental sem abrir mão de todo aparato de interdição. E, ao mesmo tempo, manter o subsídio público aos hospitais privados diretamente ou indiretamente, através do desconto do imposto de renda para os gastos em saúde, aí incluída a saúde mental, aos economicamente mais bem colocados na escala das classes sociais.

Por outro lado, os CAPS são ainda, até porque apresentam-se teórica e politicamente em disputa, locais privilegiados para implantação de um sistema de saúde que dê conta das singularidades, das especificidades individuais e acabe, se não capitular diante da intervenção do neoliberalismo, norteando um novo modelo geral de saúde que troca a universalidade pela integralidade, a coletividade pela singularidade, desterritorializando o sistema, ao mesmo tempo em que seja adequado à coletividade onde esteja.

\section{REFERÊNCIAS}

1. Organização Mundial da Saúde. Relatório sobre a Saúde no Mundo 2001. Saúde mental: nova concepção, nova esperança. Geneva (SW): OMS; 2001.

2. Murray CJL, Lopez AD. The Global Burden of Disease: a comprehensive assessment of mortality and disability from diseases, injuries and risk factors in 1990 and projected to 2020) Cambridge (US): Harvard School of Public Health; 1996.

3. Paim JS, Teixeira CF. Política, planejamento e gestão em saúde: balanço do estado da arte. Rev Saúde Pública. 2006 Ago, 40(Esp):73-8.

4. Vechi LG. Iatrogenia e exclusão social: a loucura como objeto do discurso científico no Brasil. Estud psicol (Natal). 2004 Set-Dez; 9(3):489-95.

5. Costa-Rosa A. O modo psicossocial: um paradigma das práticas substitutivas ao modo asilar. In: Amarante P, organizador. Ensaios: subjetividade, saúde mental, sociedade. Rio de Janeiro (RJ): Fiocruz; 2000. 
6. Minayo MCS. O desafio do conhecimento: pesquisa qualitativa em saúde. São Paulo/Rio de Janeiro (SP/ RJ): Hucitec/Abrasco; 1993.

7. Ministério da Saúde (BR), Secretaria de Assistência a Saúde. Portaria SAS No 147, de 25 de agosto de 1994. Brasília (DF): MS; 1994.

8. Franco TB, Bueno WS, Merhy EE. O acolhimento e os processos de trabalho em saúde: Betim, Minas Gerais, Brasil. Cad Saúde Pública. 1999 Abr-Jun; 15(2):345-53.

9. Silva AGJ, Mascarenhas MTM. Avaliação da atenção básica em saúde sob a ótica da integralidade: aspectos conceituais e metodológicos. In: Pinheiro R, Mattos RA, organizadores. Cuidado: as fronteiras da integralidade. Rio de Janeiro (RJ): HUCITEC; 2004. p.241-57.
10. Solla JJSP. Acolhimento no sistema municipal de saúde. Rev Bras Saude Mater Infant. 2005 Out-Dez; 5(4):493-503.

11. Cecílio LCO. Modelos tecno-assistenciais em saúde: da pirâmide ao círculo, uma possibilidade a ser explorada. Cad Saúde Pública. 1997 Jul-Set; 13(3):469-78

12. Wimmer GF, Figueiredo GO. Ação coletiva para qualidade de vida: autonomia, transdisciplinaridade e intersetorialidade. Cien Saude Colet. 2006 Jan-Mar; 11(1):145-54.

13. Organização Pan-Americana de Saúde. Reorientação das práticas e serviços de saúde: conceito [on-line]. [acesso 2007 Jun 01]. Disponível em: http://www.opas.org.br/coletiva/temas. $\mathrm{cfm}$ ?id=18\&area=Conceito 